\title{
ON ASYMPTOTIC BEHAVIOUR OF CERTAIN INTEGRO-DIFFERENTIAL EQUATIONS
}

\author{
EN HAO YANG
}

\begin{abstract}
This paper deals with the asymptotic behaviour of some integro-differential equations which can be seen as perturbations of some nonlinear systems of ordinary differential equations. The main tools used here are two generalizations of the Pachpatte's integral inequality and an Alekseev type nonlinear variation of constants formula.
\end{abstract}

1. The following result extends a known integral inequality established by Pachpatte in $[2$, p. 795$]$.

Lemma. Let $u(t), a(t), f(t, s), g_{i}(t, s)$ and $h_{i}(t, s), i=1,2, \ldots, n$, be nonnegative continuous functions defined on the sets $I=[0, h)$ and $I \times I$, respectively, with their ranges in $R_{+}=[0, \infty)$. Let $a(t)$ be nondecreasing on $I$, and $f(t, s), g_{i}(t, s)$ and $h_{i}(t, s)$ be nondecreasing in $t$ for each fixed $s \in I$. Suppose that the inequality

(1) $u(t) \leqslant a(t)+\int_{0}^{t} f(t, s) u(s) d s+\sum_{i=1}^{n} \int_{0}^{t} g_{i}(t, s)\left[\int_{0}^{s} h_{i}(s, m)[u(m)]^{p} d m\right] d s$

holds for $t \in I$, where $p \in(0,1]$ is a constant. Then the following are valid:

(A) If $0<p<1$,

$$
u(t) \leqslant\left[[a(t) F(t)]^{1-p}+(1-p) \sum_{i=1}^{n} G_{i}(t) F(t) \int_{0}^{t} h_{i}(t, s) d s\right]^{1 /(1-p)}
$$

when $t \in I$.

(B) If $p=1$,

$$
u(t) \leqslant a(t) \exp \int_{0}^{t}\left[f(t, s)+\sum_{i=1}^{n} G_{i}(t) F(t) h_{i}(t, s)\right] d s
$$

when $t \in I$, where

$$
F(t)=\exp \int_{0}^{t} f(t, s) d s, \quad G_{i}(t)=\int_{0}^{t} g_{i}(t, s) d s, \quad i=1,2, \ldots, n .
$$

Proof. By our assumptions, we observe that

$$
\int_{0}^{t} g_{i}(t, s)\left[\int_{0}^{s} h_{i}(s, m)[u(m)]^{p} d m\right] d s \leqslant G_{i}(t) \int_{0}^{t} h_{i}(t, s)[u(s)]^{p} d s .
$$

Received by the editors February 15, 1983 and, in revised form, March 29, 1983.

1980 Mathematics Subject Classification. Primary 34D20, 45J05.

Key words and phrases. Integro-differential equation, integral inequality, stable in variation, asymptotic behaviour, perturbations. 
Obviously, the inequalities (2) and (3) are valid when $t=0$. Now we fix an arbitrary number $T \in(0, h)$. Then from (1) we get

$$
u(t) \leqslant a(T)+\int_{0}^{t} f(T, s) u(s) d s+\sum_{i=1}^{n} G_{i}(T) \int_{0}^{t} h_{i}(T, s)[u(s)]^{p} d s, \quad t \in[0, T] .
$$

As we shall note in Remark 1 below, we may assume here that $a(t)>0$ when $t>0$. We define

$$
L(t)=a(T)+\sum_{i=1}^{n} G_{i}(T) \int_{0}^{t} h_{i}(T, s)[u(s)]^{p} d s .
$$

Since $L(t)$ is nondecreasing on $[0, T]$, by Bellman's integral inequality (see [1]) we obtain from (4)

$$
u(t) \leqslant L(t) \exp \int_{0}^{t} f(T, s) d s \quad \text { when } t \in[0, T],
$$

and hence we have

(5) $u(t) \leqslant F(T)\left[a(T)+\sum_{i=1}^{n} \int_{0}^{t} G_{i}(T) h_{i}(T, s)[u(s)]^{p} d s\right]$ when $t \in[0, T]$.

The above inequality (5) is a special case of the Bihari inequality [1], thus when $t \in[0, T]$ we have

(6) $u(t) \leqslant\left[[a(T) F(T)]^{1-p}+(1-p) \sum_{i=1}^{n} G_{i}(T) F(T) \int_{0}^{t} h_{i}(T, s) d s\right]^{1 /(1-p)}$ if $0<p<1$ and

$$
u(t) \leqslant a(T) F(T) \exp \int_{0}^{t} \sum_{i=1}^{n} G_{i}(T) F(T) h_{i}(T, s) d s
$$

if $p=1$. Finally, set $t=T$ in (6) and (7). Since $T$ is an arbitrary number from $(0, h)$, the proof of this Lemma is complete.

REMARK 1. The restriction " $a(t)>0$ when $t>0$ ", used in the above, can be removed by adding a positive number $\varepsilon$ to the function $a(t)$ contained in (1), and then letting $\varepsilon$ tend to zero in (1), (2) and (3).

2. In this section, we consider the following integro-differential equation:

$$
y^{\prime}=f(t, y)+g\left[t, y, \int_{0}^{t} h[t, s, y(s)] d s\right], \quad t \in R_{+}, y \in R^{n} .
$$

It can be seen as a perturbation of the differential equation

$$
x^{\prime}=f(t, x), \quad t \in R_{+}, x \in R^{n},
$$

where $f: R_{+} \times R^{n} \rightarrow R^{n}$ is a continuously differentiable function and $g: R_{+} \times R^{n} \times$ $R^{n} \rightarrow R^{n}$ and $h: R_{+} \times R_{+} \times R^{n} \rightarrow R^{n}$ are continuous functions, $f(t, 0)=$ $g(t, 0,0)=h(t, s, 0) \equiv 0$.

To simplify the statement, with regard to (ND), we shall assume that the global existence and the uniqueness of its solutions are valid. But for the equation (NP) we 
shall merely assume the uniqueness and the local existence. We will denote by $x\left(t, t_{0}, \xi_{0}\right)$ and $y\left(t, t_{0}, \xi_{0}\right)$ the solutions of (ND) and (NP) passing through the point $\left(t_{0}, \xi_{0}\right)$, respectively; and for simplicity, let $R^{n}$ be the Euclidean $n$-space. We define

$$
\Phi\left(t, s, x_{0}\right)=\frac{\partial}{\partial x_{0}} x\left(t, s, x_{0}\right) .
$$

Definition 1 [4]. If there exist positive constants $\delta$ and $M=M(\delta)$ such that

$$
\left\|\Phi\left(t, t_{0}, x_{0}\right)\right\| \leqslant M \text { and }\left\|x\left(t, t_{0}, x_{0}\right)\right\| \leqslant M R\left\|x_{0}\right\| \text { for } t \geqslant t_{0} \geqslant 0
$$

hold for all $x_{0} \in R^{n}$ with $\left\|x_{0}\right\| \leqslant \delta$ and for every $t_{0} \geqslant 0$, then we say that the trivial solution $x(t) \equiv 0$ of (ND) is locally and uniformly stable in variation. Moreover, if $\delta$ can be chosen $\delta \rightarrow \infty$ then the above stability is said to be global.

Definition 2 [5]. If there exist positive constants $c$ and $\delta$, and $M=M(\delta)$ such that

$$
\begin{aligned}
\left\|\Phi\left(t, t_{0}, x_{0}\right)\right\| & \leqslant M \exp \left[-c\left(t-t_{0}\right)\right], \\
\left\|x\left(t, t_{0}, x_{0}\right)\right\| & \leqslant M\left\|x_{0}\right\| \exp \left[-c\left(t-t_{0}\right)\right], \quad \text { when } t \geqslant t_{0} \geqslant 0
\end{aligned}
$$

hold for all $x_{0} \in R^{n}$ with $\left\|x_{0}\right\| \leqslant \delta$ and for every $t_{0} \geqslant 0$, then we say that the trivial solution of (ND) is uniformly and exponentially stable in variation with the exponent $c$. Further, if $\delta$ can be chosen $\delta \rightarrow \infty$ then this stability is said to be global.

We note that on our assumptions the following nonlinear variation of constants formula $(\mathrm{CV})$ is true. It can be proved by taking $t_{0}=0$ and following the same argument as in the proof of Lemma 3 in Brauer [3].

(CV) $y(t)-x\left(t, 0, \xi_{0}\right)=\int_{0}^{t} \Phi(t, s, y(s)) g\left[s, y(s), \int_{0}^{s} h[s, m, y(m)] d m\right] d s$

when $t \in I\left(\xi_{0}\right), \xi_{0} \in R^{n}$, where $y(t)=y\left(t, 0, \xi_{0}\right)$ and $I\left(\xi_{0}\right)$ denotes the maximal interval on which the solution $y(t)$ is existing.

THEOREM 1. In addition to above assumptions on (ND) and (NP), we assume the following:

(1) For $t \geqslant s \geqslant 0$ and $y \in R^{n}$ we have

$$
\|h[t, s, y]\| \leqslant H(t, s)\|y\|^{p}+e_{1}(t)+e_{2}(s)
$$

where $p \in(0,1]$ is a constant; $e_{1}, e_{2}: R_{+} \rightarrow R_{+}$and $H: R_{+} \times R_{+} \rightarrow R_{+}$are continuous functions, and $H(t, s)$ is nondecreasing in $t$ for each $s$ fixed.

(2) For $t \geqslant 0, y \in R^{n}$ and $z \in R^{n}$ we have

$$
\|g(t, y, z)\| \leqslant p(t)\|y\|+q(t)\|z\|+e_{3}(t)
$$

where $p(t), q(t)$ and $e_{3}(t): R_{+} \rightarrow R_{+}$are continuous functions.

(3) The trivial solution of (ND) is globally (or locally) uniformly stable in variation.

(4) The functions $p(t), q(t)$ and $e_{3}(t)$ are in $L_{1}(0, \infty)$.

(5) When $t \rightarrow \infty$ we have $\int_{0}^{t} H(t, s) d s<\infty$, and

$$
\int_{0}^{t} q(s)\left[\int_{0}^{s}\left[e_{1}(s)+e_{2}(m)\right] d m\right] d s<\infty .
$$


Then all solutions $y\left(t, 0, \xi_{0}\right)$ (or for all $\xi_{0} \in R^{n}$ with $\left\|\xi_{0}\right\|$ sufficiently small) of the equation (NP) are existing on $R_{+}$and are bounded.

Proof. According to Definition 1, by hypothesis (3) we have

$$
\left\|x\left(t, 0, \xi_{0}\right)\right\| \leqslant M\left\|\xi_{0}\right\|, \quad\left\|\Phi\left(t, s, y\left(s, 0, \xi_{0}\right)\right)\right\| \leqslant M
$$

when $t \geqslant s \geqslant 0$ and $\xi_{0} \in R^{n}$ (or $\left\|\xi_{0}\right\| \leqslant \delta$ ), so by the nonlinear variation of constants formula (CV) and in view of the hypotheses (1), (2) and (3), we can derive

$$
\begin{aligned}
\left\|y\left(t, 0, \xi_{0}\right)\right\| \leqslant & U\left(t, \xi_{0}\right)+\int_{0}^{t} M p(s)\left\|y\left(s, 0, \xi_{0}\right)\right\| d s \\
& +\int_{0}^{t} M q(s)\left[\int_{0}^{s} H(s, m)\left\|y\left(m, 0, \xi_{0}\right)\right\|^{p} d m\right] d s
\end{aligned}
$$

for $t \in I\left(\xi_{0}\right), \xi_{0} \in R^{n}$ (or $\left.\left\|\xi_{0}\right\| \leqslant \delta\right)$, where

(9) $U\left(t, \xi_{0}\right)=M\left\|\xi_{0}\right\|+\int_{0}^{t} M e_{3}(s) d s+\int_{0}^{t} M q(s)\left[\int_{0}^{s}\left[e_{1}(s)+e_{2}(m)\right] d m\right] d s$.

An application of the Lemma to the above inequality yields the following.

(A) If $0<p<1$,

$$
\left\|y\left(t, 0, \xi_{0}\right)\right\| \leqslant\left[\left[U\left(t, \xi_{0}\right) P(t)\right]^{1-p}+(1-p) P(t) Q(t) \int_{0}^{t} H(t, s) d s\right]^{1 /(1-p)}
$$

for $t \in I\left(\xi_{0}\right), \xi_{0} \in R^{n}$ (or $\left\|\xi_{0}\right\| \leqslant \delta$ ), where $P(t)=\exp \int_{0}^{t} M p(s) d s$ and $Q(t)=$ $\int_{0}^{t} M q(s) d s$.

(B) If $p=1$,

$$
\left\|y\left(t, 0, \xi_{0}\right)\right\| \leqslant U\left(t, \xi_{0}\right) \exp \int_{0}^{t}[M p(s)+P(t) Q(t) H(t, s)] d s
$$

for $t \in I\left(\xi_{0}\right), \xi_{0} \in R^{n}$ (or $\left\|\xi_{0}\right\| \leqslant \delta$ ). Using hypotheses (4) and (5), we can obtain the global existence and the boundedness of $y\left(t, 0, \xi_{0}\right)$ from (9), (10) and (11). Because this is a standard argument we omit the details. The proof of this theorem is now completed.

Corollary 1. If in Theorem 1 we also have the conditions $p=1$ and $e_{i}(t)=0$ $(i=1,2,3)$, then the trivial solution $y(t) \equiv 0$ of the equation (NP) is stable in the sense of Liapunov.

EXAMPLE 1. Consider the integro-differential equation

$$
y^{\prime}=-\frac{1}{2} y^{3}+e^{-t} \sin t \int_{0}^{t} h(s)[y(s)]^{p} d s, \quad t \in R_{+}, y \in R,
$$

where $0<p \leqslant 1$ is a constant. We can easily obtain that the general solution of

$$
x^{\prime}=-\frac{1}{2} x^{3}, \quad t \in R_{+}, x \in R,
$$

is in the form

$$
x\left(t, t_{0}, \xi_{0}\right)=\xi_{0}\left[\xi_{0}^{2}\left(t-t_{0}\right)+1\right]^{-1 / 2}, \quad \xi_{0} \in R, t \geqslant t_{0} \geqslant 0,
$$


and hence

$$
\Phi\left(t, t_{0}, \xi_{0}\right)=\frac{\partial}{\partial \xi_{0}} x\left(t, t_{0}, \xi_{0}\right)=\left[\xi_{0}^{2}\left(t-t_{0}\right)+1\right]^{-3 / 2}
$$

Therefore,

$$
\left\|x\left(t, t_{0}, \xi_{0}\right)\right\| \leqslant\left\|\xi_{0}\right\|, \quad\left\|\Phi\left(t, t_{0}, \xi_{0}\right)\right\| \leqslant 1,
$$

when $\xi_{0} \in R^{n}, t \geqslant t_{0} \geqslant 0$. Thus the trivial solution of (13) is globally uniformly stable in variation, so by Corollary 1 and Theorem 1 , if $h(t): R_{+} \rightarrow R$ is a continuous function with $|h(t)| \in L_{1}(0, \infty)$, then all of the solutions of the integrodifferential equation (12) are defined and bounded on the whole $R_{+}$, and if $p=1$ then the trivial solution of (12) is stable in the sense of Liapunov.

THEOREM 2. Suppose that hypotheses (1) and (2) of Theorem 1 are satisfied, and we assume further that:

3. The trivial solution of (ND) is globally, uniformly and exponentially stable in variation with the exponent $c>0$.

4. The functions $p(t), q(t) e^{c t}$ and $e_{3}(t) e^{c t}$ are in $L_{1}(0, \infty)$.

5. When $t \rightarrow \infty$ we have

$$
\int_{0}^{t} H(t, s) e^{-p c s} d s<\infty, \quad \int_{0}^{t} q(s) e^{c s}\left[\int_{0}^{s}\left[e_{1}(s)+e_{2}(m)\right] d m\right] d s<\infty .
$$

Then all solutions (or, for all $\xi_{0} \in R^{n}$ with $\left\|\xi_{0}\right\|$ sufficiently small) of the equation (NP) can be prolonged on the whole $R_{+}$and obey the relation

$$
\left\|y\left(t, 0, \xi_{0}\right)\right\|=O[\exp (-c t)] \text { as } t \rightarrow \infty \text {. }
$$

Proof. By following an argument similar to that used in the proof of Theorem 1, and by using Definition 2, we easily obtain

$$
\begin{aligned}
z(t) \leqslant & V\left(t, \xi_{0}\right)+\int_{0}^{t} M p(s) z(s) d s \\
& +\int_{0}^{t} M q(s) e^{c s}\left[\int_{0}^{s} H(s, m) e^{-p c m}[z(m)]^{p} d m\right] d s
\end{aligned}
$$

for $\xi_{0} \in R^{n}\left(\right.$ or $\left\|\xi_{0}\right\| \leqslant \delta$ ) and $t \in I\left(\xi_{0}\right)$, where

$$
\begin{gathered}
V\left(t, \xi_{0}\right)=M\left\|\xi_{0}\right\|+\int_{0}^{t} M e_{3}(s) e^{c s} d s+\int_{0}^{t} M q(s) e^{c s}\left[\int_{0}^{s}\left[e_{1}(s)+e_{2}(m)\right] d m\right] d s, \\
z(t)=e^{c t}\left\|y\left(t, 0, \xi_{0}\right)\right\| .
\end{gathered}
$$

So by our Lemma and (14) we get the following:

(A) If $0<p<1$,

(15) $z(t) \leqslant\left[\left[V\left(t, \xi_{0}\right) P(t)\right]^{1-p}+(1-p) P(t) Q_{1}(t) \int_{0}^{t} H(t, s) e^{-p c s} d s\right]^{1 /(1-p)}$

for $\xi_{0} \in R^{n}$ (or $\left\|\xi_{0}\right\| \leqslant \delta$ ) and $t \in I\left(\xi_{0}\right)$, where $P(t)=\exp \int_{0}^{t} M p(s) d s$ and $Q(t)=$ $\int_{0}^{t} M q(s) e^{c s} d s$. 
(B) If $p=1$,

$$
z(t) \leqslant V\left(t, \xi_{0}\right) \exp \int_{0}^{t}\left[M p(s)+P(t) Q_{1}(t) e^{-p c s} H(t, s)\right] d s,
$$

for $\xi_{0} \in R^{n}$ (or $\left\|\xi_{0}\right\| \leqslant \delta$ ) and $t \in I\left(\xi_{0}\right)$.

The remainder of the argument is elementary, so we omit the details.

Corollary 2. If in Theorem 2 we also have $p=1, e_{i}(t) \equiv 0(i=1,2,3)$, then the trivial solution of the integro-differential equation (NP) is exponentially stable in the sense of Liapunov.

EXAMPLE 2. We consider the equation

$$
y^{\prime}=(\cos t-3) y+e^{-3 t} \int_{0}^{t} h(s)[y(s)]^{q} d s, \quad t \in R_{+}, y \in R .
$$

Here $q \in(0,1]$ is a constant. It is easy to see that the trivial solution of the unperturbed linear differential equation

$$
x^{\prime}=(\cos t-3) x, \quad t \in R_{+}, x \in R,
$$

is globally and uniformly, exponentially stable in variation with an exponent $c=2$. So by Theorem 2, if the function $h: R_{+} \rightarrow R$ is a continuous function with $|h(t)| e^{-2 q t} \in L_{1}(0, \infty)$, then all solutions of the equation (17) exist on the whole $R_{+}$ and obey

$$
\|y(t)\|=O[\exp (-2 t)] \quad \text { as } t \rightarrow \infty .
$$

In addition, if $q=1$ then by Corollary 2 the trivial solution $y(t) \equiv 0$ of (17) is exponentially stable in the sense of Liapunov.

The author wishes to thank Professor George R. Sell for his helpful suggestions.

\section{REFERENCES}

1. I. Bihari, A generalization of a lemma of Bellman and its application to uniqueness problems of differential equations, Acta Math. Acad. Sci. Hungar. 7 (1956), 81-84.

2. B. G. Pachpatte, On some integral inequalities similar to Bellman-Bihari type, J. Math. Anal. Appl. 49 (1975), 794-802.

3. F. Brauer, Perturbations of nonlinear sy'stems of differential equations, J. Math. Anal. Appl. 14 (1966), 198-206.

4. F. Brauer and A. Strauss, Perturbations of nonlinear systems of differential equations. III, J. Math. Anal. Appl. 31 (1970), 37-48.

5. B. G. Pachpatte, Perturbations of nonlinear systems of differential equations, J. Math. Anal. Appl. 51 (1975), 550-556.

Department of Mathematics, Jinan University, Guang Zhou, China 